\title{
Effect of Initial Deflection of Diamond Wire on Thickness Variation of Sapphire Wafer in Multi-Wire Saw
}

\author{
Doyeon Kim,2, Hyoungjae Kim, Sangjik Lee', and Haedo Jeong ${ }^{2, \#}$ \\ 1 Korea Institute of Industrial Technology, 30, Gwahaksandan 1-ro 60beon-gil, Gangseo-gu, Busan, 618-230, South Korea \\ 2 Graduate School of Mechanical Engineering, Pusan National University, 2, Busandaehak-ro 63beon-gil, Geumjeong-gu, Busan, 609-836, South Korea \\ \# Corresponding Author / E-mail: hdjeong@pusan.ac.kr, TEL: +82-51-510-2463, FAX: +82-51-518-8442
}

KEYWORDS: Diamond wire, Multi wire saw, Sapphire wafer, Total thickness variation, Wire deflection

\begin{abstract}
Sapphire wafers are widely used as substrates for fabricating gallium nitride light-emitting diodes. The quality of light-emitting diodes depends on the total thickness variation and BOW of the wafers. The multi-wire saw process is critical in determining post-processing times for steps such as diamond mechanical polishing and chemical mechanical polishing. In particular, thickness variation is affected by wear of diamond wires, which in turn is controlled by cutting conditions including wire speed, feed rate, initial contact condition between ingot and wire, and new wire consumption. Thickness variation shows a marked change from the initial to final feeding location during the multi-wire sawing process. A wire is not worn when it is initially contacted by an ingot. Hence, the initial kerf loss is greater than the kerf loss at the final feeding location. This study focused on minimizing the thickness variation between the initial and final contact points of the wafer. Experiments were conducted with different initial wire deflection conditions. The experimental results showed that increasing the wire deflection increased the cutting load, which in turn caused severe wear of the wire. Consequently, the thickness variation of the wafer in the multi-wire sawing process was controlled by adjusting the wire deflection
\end{abstract}

\section{Introduction}

Sapphire substrates are suitable for use in light-emitting diode (LED) and non-LED applications owing to their high strength, high temperature resistance, good electrical insulation, and low dielectric loss. The sapphire substrate industry is anticipated to grow because of the influx of non-LED applications such as smart phones, camera lens covers, and smart watches. ${ }^{1}$ However, the production cost of wafers should be constantly reduced to ensure the competitive pricing of substrates and to reduce the final LED production cost. ${ }^{2}$

Multi-wire sawing is one of the critical processes that determine the accuracy of the wafer shape and subsequent processing times. In particular, the diamond wire is the most important component in multiwire sawing and crucially affects the accuracy of the wafer shape. The greatest advantages of using diamond wire in multi-wire sawing are the low kerf loss, minimal surface damage, and high productivity. ${ }^{3}$

A recent study on diamond wire was conducted to analyze the effect of varying the manufacturing method on the cutting characteristics of the obtained wire. ${ }^{4,5}$ However, the study did not sufficiently analyze the actual states caused by mass production and identify the solutions.
In particular, multi-wire sawing is the first process that determines the accuracy of wafer shape in the wafering process. The degree of shape accuracy achieved during this process in turn determines the entire processing time and total cost.

Fig. 1 shows the thickness variation of a wafer across the feeding direction when new diamond wires are used in the multi-wire sawing process. The wafer is thinner around the inlet position of the wire than around the outlet position where the cutting has finished. This result can be explained in accordance with the causes leading to thickness variation of wafers, which were discussed in previous studies. ${ }^{6,7}$

The thickness variation of a wafer is caused by variation in the diameter of the diamond wire due to wear. Owing to high kerf loss, the initial part that contacts the ingot and wire is thinner than the end part, at which cutting by the new wire is completed.

This thickness variation affects the overall thickness variation of the wafer.

This is the main reason why the processing time and production cost increase, because the thickness variation must be removed by postprocessing. Ultimately, the critical factor that determines the thickness variation of the wafer is the variation formed by initial point and end point. 


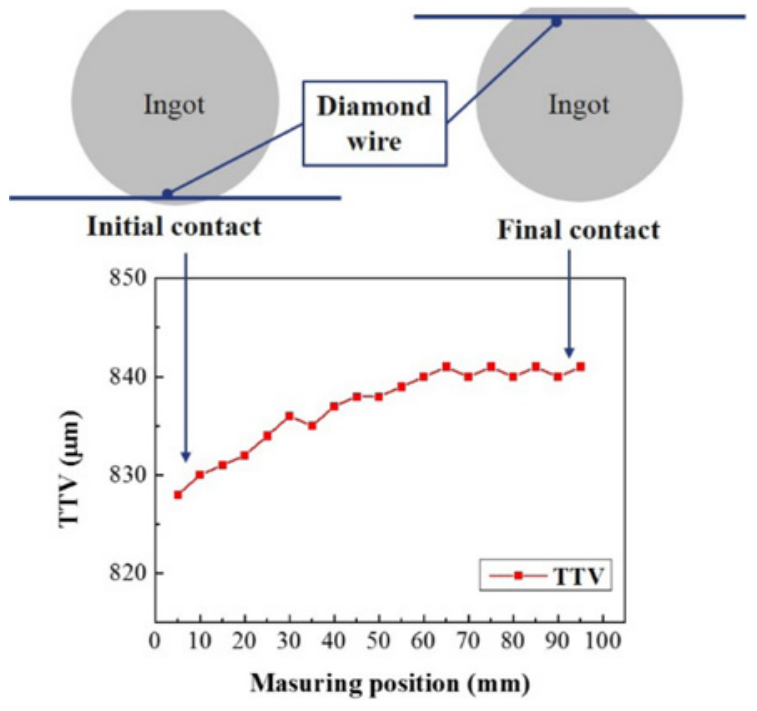

Fig. 1 Thickness variation of 4-inch sapphire wafer

The main purpose of this study is to analyze how initial wire deflection affects the process and characteristics of the initial thickness variation of the wafer.

\section{Experimental Setup}

The main parameters of the experiments are summarized in Table 1, which includes the feed speed of the ingot, initial wire deflection, and type of diamond wire. Experiments were performed using a single wire saw machine equipped with two $5 \mathrm{~kg}$ capacity load cells for measuring the cutting force acting along the wire moving direction and the cutting load acting against the feeding direction. (Fig. 2(a)) The diameter of the diamond wire was measured after every 100th contact between the ingot and wire at different ingot feed rates.

The ingot used in this experiment had a square shape $(50 \mathrm{~mm} \times 50 \mathrm{~mm})$, which was cut along the C-plane. The commercial Ni-electroplated diamond wire tool used in this experiment had diamond abrasives 30 to $40 \mu \mathrm{m}$ in diameter and a core wire with a diameter of $0.18 \mathrm{~mm}$.

The variation in diameter of the diamond wire was measured using vision system shown in Fig. 2(b).

This system consists of a light source, a CCD camera, and a computer with an image processing system. A high-resolution $\mathrm{CCD}$ camera, with a resolution of $1,600 \times 1,200$ pixels, was used to characterize the diameter of the wire after a given number of reciprocating motions. The CCD camera was installed parallel to the light source to capture the image of the diamond wire. The captured image was converted to a black-and-white type binary image to obtain the height of the diamond abrasive and diameter of the diamond wire.

The kerf loss of the sapphire ingot was measured by a digital microscope (VHX-2000X, Keyence). The width of the cutting path in the ingot was measured along the feed direction by the microscope to characterize the kerf loss. The acquired image was also converted to a binary image to enhance cutting edges.

To observe the variation in the diameter of the diamond wire after the experiment, $1 \mathrm{~mm}$ sections were measured every $540 \mathrm{~mm}$ of the wire. Fifty measurements were obtained for each experiment and the average diameter was calculated from the vision measurement results.
Table 1 Experimental conditions

\begin{tabular}{cc}
\hline Parameter & Condition \\
\hline Wire tension & $40 \mathrm{~N}$ \\
\hline Wire speed & $400 \mathrm{~m} / \mathrm{min}$ \\
\hline Ingot feeding speed & $0.75 \mathrm{~mm} / \mathrm{min}$ \\
\hline Type of nickel plated diamond wire & Core $0.18 \mathrm{~mm}, 30 \sim 40 \mu \mathrm{m} \mathrm{dia.}$ \\
\hline Initial wire deflection & $0,2,4 \mathrm{~mm}$ \\
\hline No. of contact between ingot and wire & $100,200,300$ \\
\hline Workpiece & $50 \mathrm{~mm}$ square \\
& C-plane sapphire ingot \\
\hline Coolant & DKW-1P, $5 \mathrm{wt} \%$ diluted \\
\hline
\end{tabular}

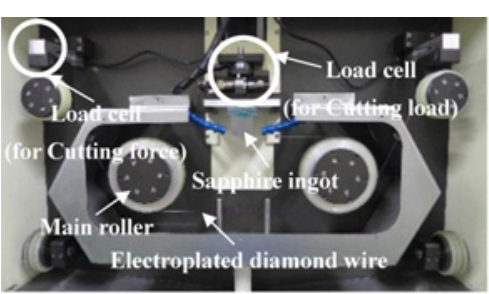

(a) Single wire saw

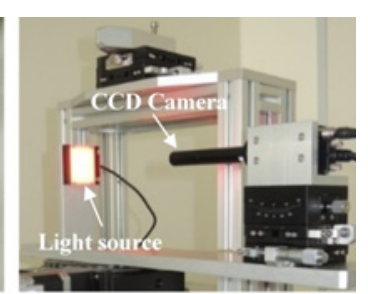

(b) Vision system
Fig. 2 Experimental equipment

\section{Results and Discussion}

\subsection{Effect of Wire Deflection on Cutting Ability}

The wire deflection is proportional to the ingot feed rate and the contact length between the diamond wire and ingot, but inversely proportional to the wire tension. ${ }^{8}$

Fig. 3 shows the results of force signals during the cutting process with different initial wire deflections. D0, D2, and D4 represent the amounts of initial deflection at the beginning of the cutting process, which were set to 0,2 , and $4 \mathrm{~mm}$, respectively.

The loadcell signal in the D0 condition shows that the cutting load sharply increased near the initial stage of cutting but its slope gradually decreased with cutting time. In the case of D2, the cutting load also sharply increased but the load signal promptly stabilized within a shorter time than in the D0 condition. In the case of D4, the loadcell signal shows that the cutting load temporarily increased at the start of cutting, deceased gradually, and finally became stable.

The cutting load is linearly proportional to the wire deflection, which results from subtracting the penetration speed of the wire into the ingot from the feeding speed of the ingot. ${ }^{9}$ The D0 case shows that the penetration speed of the wire into the ingot is slower than in the D2 or D4 cases owing to the wire deflection.

Therefore, the contact time with the wire at the initial cutting stage is longer in the case of D0.

The cutting force along the wire moving direction is proportional to the cutting load and contact length between the ingot and wire. In the case of D0, the cutting force starts from nearly zero because the cutting load starts at zero. In the case of D2 and D4, the cutting force is proportional to the initial deflection of the wire. All three cases show a break-in procedure at the first stage of the cutting process and become stable after $1000 \mathrm{~s}$.

These results imply that the initial cutting phenomenon is affected by the initial deflection of the wire. Therefore, the residence time of the wire with the ingot varies with the ingot feeding position even under 


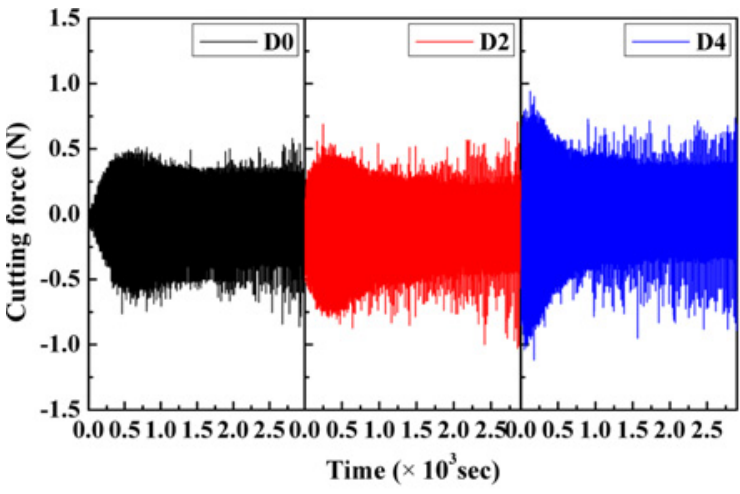

(a) Cutting force

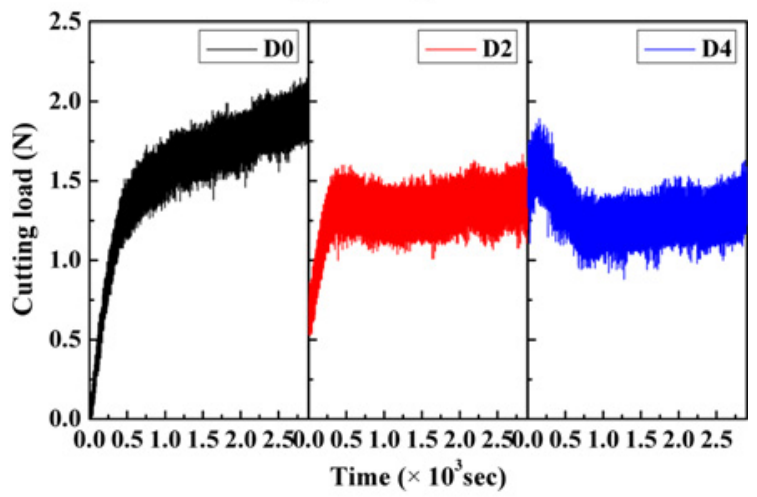

(b) Cutting load

Fig. 3 Cutting ability of wire with 300 contacts between wire and ingot

the same feed speed when the initial wire deflections differ.

\subsection{Effect of Diamond Abrasive Wear on Wire Deflection}

During the diamond wire sawing process, the wires move back and forth to achieve better cutting performance and more effective use of the diamond abrasives. The reciprocating motion results in gradual wear of the wires. The average extrusion height of diamond abrasives measured by the vision system is shown in Fig. 4 for different initial wire deflections. When the number of contacts between the ingot and wire is 100 , the average extrusion height is lower for D2 and D4 than for D0.

In particular, the results show that if the number of contacts is 200 , the difference in average extrusion height between D2 or D4 and D0 widens greatly. However, the difference gradually decreases if the number of contacts is 300 .

This phenomenon is explained in this case by Archard's wear law. As is described by the equation, ${ }^{10,11}$ the amount of wear (V) of an abrasive is linearly proportional to the normal load (L) and sliding distance $(\mathrm{S})$ but inversely proportional to its hardness $(\mathrm{H})$. That is,

$$
V \propto \frac{L S}{H} \text { or } V=K \frac{L S}{H}
$$

where $\mathrm{K}$ is a dimensionless constant known as the wear coefficient. ${ }^{12}$ According to Archard's wear law, the wear amount of a wire is determined by the sliding distance and cutting load. In this experiment, all conditions were identical except for the load.

The results of cutting force and cutting load show that D2 and D4 have a higher starting load than D0. This is explained by Eq. (1)

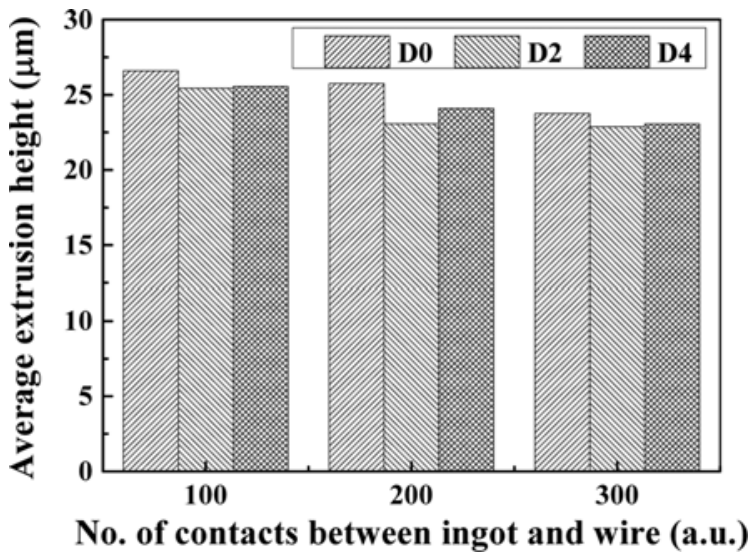

Fig. 4 Average extrusion height of diamond abrasive

because the higher initial cutting load accelerates the wear rate of the diamond abrasive. Therefore, D0 has a high average extrusion height.

The characteristics of abrasive wear in D2 and D4 show that rapid wear of the abrasive occurs between 100 and 200 contacts; however, the gap is smaller if the number of contacts is 300 . These results indicate that the initial wire deflection results in a higher cutting load in the range of 100 to 200 contacts between the wire and the ingot.

\subsection{Effect of Processing Characteristics on Wire Deflection}

The initial deflection of a wire affects abrasive wear, which determines the cutting ability of the wire. Because the wear of a diamond wire determines its nominal diameter, this wear is a critical factor that affects the total kerf loss. Fig. 5 shows the extrusion height distribution of abrasives with different numbers of contact for each wire deflection. The wear rate increases not only with the number of contacts but also with the amount of wire deflection.

The break-in time for diamond wire in cases D2 and D4 seems to be faster than in case D0 (Figs. 3 and 5).

In particular, abrasives with a height of $25-35 \mu \mathrm{m}$ on new wire become worn faster when the number of contacts between the ingot and wire is increased.

That is, the highest point of diamond abrasives, which has the greatest impact on kerf loss, is reduced by wear due to the initial wire deflection. This is also shown in the kerf loss result in the initial cutting position of Fig. 6 . As the starting wire deflection increases, the amount of kerf loss decreases as because of fast abrasive wear in the initial cutting position of $0-4 \mathrm{~mm}$. A combined effect apparently results in smaller kerf loss at higher initial deflections.

The greater the initial wire deflection, the faster the wear of abrasives on the wire. The other reason is that the greater the initial wire deflection, the higher the initial cutting speed.

The removal speed of the nickel layer surrounding the diamond abrasives is affected by wire deflection, which causes variations in the cutting ability of a wire. In the absence of any deflection, the wire cutting ability depends on the number of contacts between the ingot and wire and the ingot feed rate. However, if the wire is initially deflected, the cutting ability of the wire depends not only on the number of contacts but also on the initial cutting load by wire deflection.

In the presence of an initial wire deflection, the cutting depth 


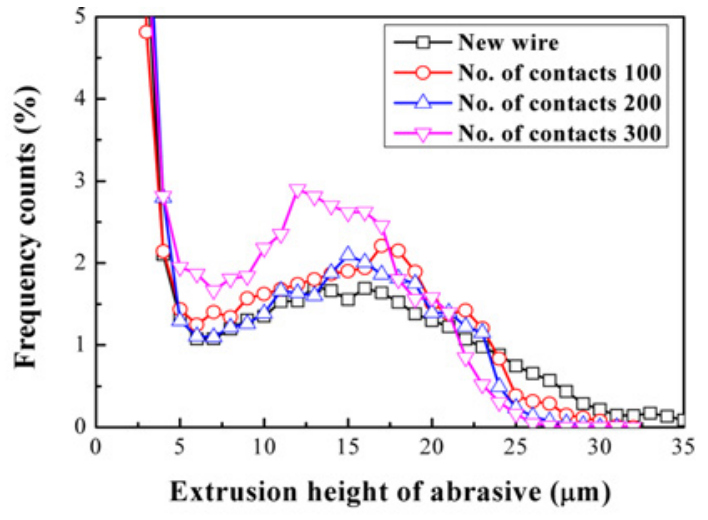

(a) Wire deflection: $0 \mathrm{~mm}$

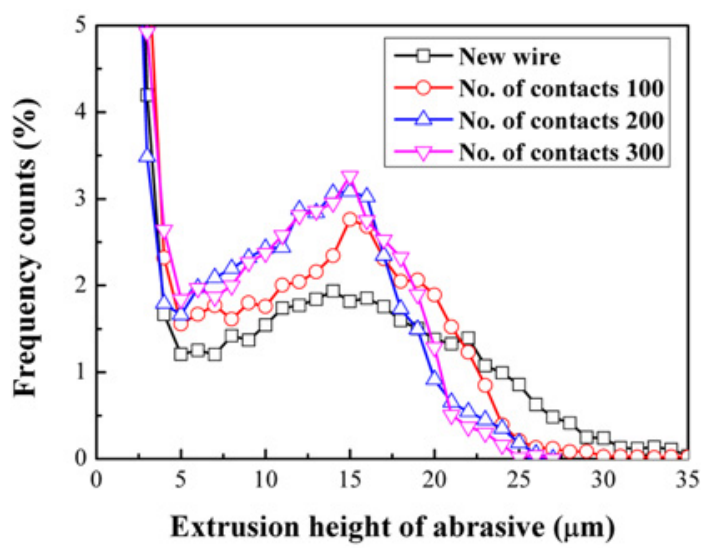

(b) Wire deflection: $2 \mathrm{~mm}$

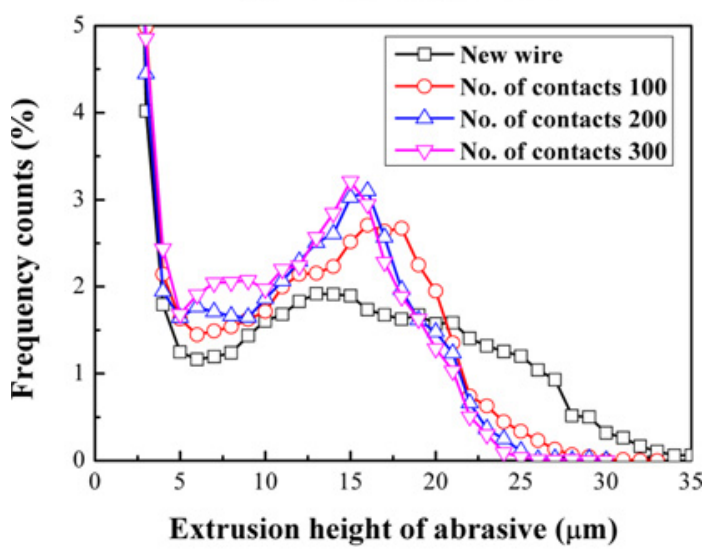

(c) Wire deflection: $4 \mathrm{~mm}$

Fig. 5 Histogram of extrusion height of diamond grit

increases owing to the higher cutting load. As shown in Fig. 7, the cutting depth is predominantly affected by the initial wire deflection and number of contacts between the ingot and diamond wire. The cutting depth was less than the target value $(30 \mathrm{~mm})$ in the case of D0, but almost the same as the target feed depth in the case of D2 and D4. This result suggests that the gradual deflection of wire during ingot feeding resulted in insufficient cutting depth, and that the smaller contact force between the wire and ingot led to insufficient in-process dressing in the D0 condition. However, the dressing efficiency was higher in cases D2 and D4. Therefore, the final cutting depth is higher for the D2 and D4 cases than for the D0 case.

This is presumably because the initial wire deflection causes the diamond abrasive to wear rapidly.

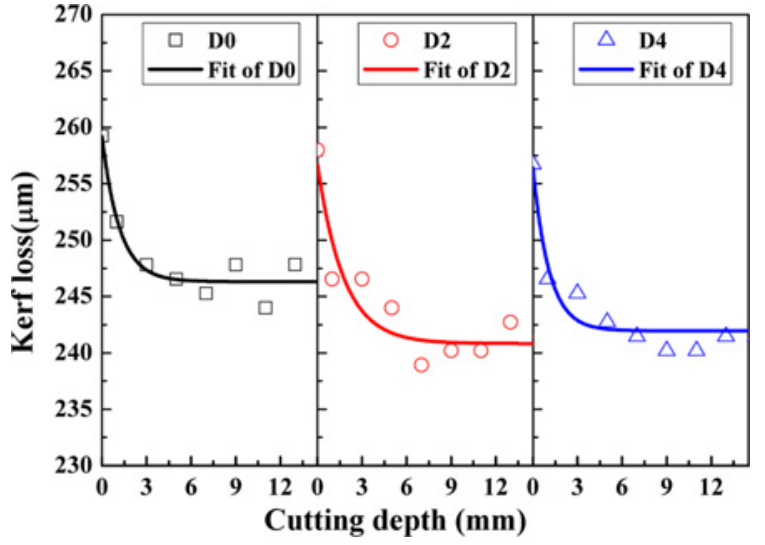

Fig. 6 Results of kerf loss

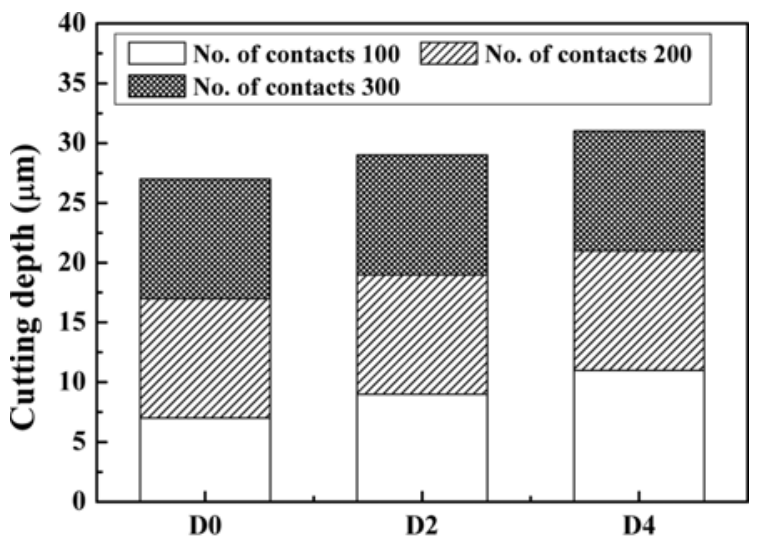

Wire deflection

Fig. 7 Cutting depth

The increased rate of initial abrasive wear is made high the cutting ability of wire which is caused by decreasing kerf loss and shortening break-in time.

\section{Conclusions}

This study focused on analyzing the effect of initial wire deflection on the thickness variation of a wafer. Experiments were conducted to determine how the initial wire deflection affects the extrusion height of diamond abrasives and cutting performance. The results showed that the wear rate of wire increased as the initial deflection increased. This is due to increased force on abrasives at a contact point. Because the wear rate of diamond grit is the most important parameter that determines the diameter of a diamond wire, the kerf loss is also influenced by wear of the wire. In addition, the cutting speed around the initial contact region affects the kerf loss of ingot materials. Therefore, the thickness variation across the wafer is strongly affected by the cutting speed and diamond wear. These parameters can be controlled by adjusting the initial wire deflection.

\section{REFERENCES}

1. LEDinside, "Sapphire Substrate to Dominate LED Market in 2014," http://www.ledinside.com (Accessed 26 January 2015) 
2. Lee, H., Park, Y., Lee, S., and Jeong, H., "Effect of Wafer Size on Material Removal Rate and Its Distribution in Chemical Mechanical Polishing of Silicon Dioxide Film," Journal of Mechanical Science and Technology, Vol. 27, No. 10, pp. 2911-2916, 2013.

3. Teomete, E., "Roughness Damage Evolution due to Wire Saw Process,” Int. J. Precis. Eng. Manuf., Vol. 12, No. 6, pp. 941-947, 2011.

4. Kim, H., Kim, D., Kim, C., and Jeong, H., "Multi-Wire Sawing of Sapphire Crystals with Reciprocating Motion of Electroplated Diamond Wires," CIRP Annals-Manufacturing Technology, Vol. 62, No. 1, pp. 335-338, 2013.

5. Chung, C. and Nhat, N. V., "Generation of Diamond Wire Sliced Wafer Surface based on the Distribution of Diamond Grits," Int. J. Precis. Eng. Manuf., Vol. 15, No. 5, pp. 789-796, 2014.

6. Chiba, Y., Tani, Y., Enomoto, T., and Sato, H., "Development of a High-Speed Manufacturing Method for Electroplated Diamond Wire Tools," CIRP Annals-Manufacturing Technology, Vol. 52, No. 1, pp. 281-284, 2003.

7. Kim, H., Kim, D., Kim, C., and Jeong, H., "Multi-Wire Sawing of Sapphire Crystals with Reciprocating Motion of Electroplated Diamond Wires," CIRP Annals-Manufacturing Technology, Vol. 62, No. 1, pp. 335-338, 2013.

8. Clark, W. I., Shih, A. J., Hardin, C. W., Lemaster, R. L., and McSpadden., S. B., "Fixed Abrasive Diamond Wire Machining-Part I: Process Monitoring and Wire Tension Force, International Journal of Machine Tools \& Manufacture, Vol. 43, pp. 523-532, 2003.

9. Lee, S., Kim, H., Kim, D., and Park, C., "Investigation on Diamond Wire Break-in and Its Effects on Cutting Performance in Multi-Wire Sawing, Proc. of the Asian Society for Precision Engineering and Nanotechnology, 2013.

10. Ludema, K. C., "Friction, Wear, Lubrication: A Textbook in Tribology," CRC Press, 1996.

11. Liu, R. and Li, D., "Modification of Archard's Equation by Taking Account of Elastic/Pseudoelastic Properties of Materials," Wear, Vol. 251, No. 1, pp. 956-964, 2001.

12. Suh, N. P., "Tribophysics," Pretice-Hall, 1986. 